\title{
Polymerization Shrinkage and Degree of Conversion of New Zirconia-Reinforced Rice Husk Nanohybrid Composite
}

\author{
Galvin Sim Siang Lin ${ }^{1} \quad$ Nik Rozainah Nik Abdul Ghani ${ }^{1} \quad$ Noor Huda Ismail ${ }^{2}$ \\ Kiran Prabhakar Singbal ${ }^{3}$ Noraida Mamat Mohd Yusuff ${ }^{4}$
}

${ }^{1}$ Conservative Dentistry Unit, School of Dental Sciences, Universiti Sains Malaysia, Kubang Kerian, Kota Bharu, Kelantan, Malaysia

2Prosthodontic Unit, School of Dental Sciences, Universiti Sains Malaysia, Kubang Kerian, Kota Bharu, Kelantan, Malaysia

${ }^{3}$ Department of Restorative Dentistry, Faculty of Dentistry,

Mahsa University, Selangor, Malaysia

${ }^{4}$ Paediatric Dentistry Unit, School of Dental Sciences, Universiti

Sains Malaysia, Kubang Kerian, Kota Bharu, Kelantan, Malaysia

Eur J Dent:2020;14:448-455

\begin{abstract}
Address for correspondence Nik Rozainah Nik Abdul Ghani, BDS (Malaya), MScClinical (Endodontic) Conservative Dentistry Unit, School of Dental Sciences, Universiti Sains Malaysia, Health campus, 16150, Kubang Kerian, Kelantan, Malaysia (e-mail: rozainah@usm.my).
\end{abstract}

\begin{abstract}
Keywords

- composite resin

- polymerization shrinkage

- degree of conversion

- rice husk

- zirconia
\end{abstract}

Objectives This study aimed to compare the polymerization shrinkage and degree of conversion of new zirconia-reinforced rice husk nanohybrid composite with commercialized microhybrid and nanofilled composites.

Materials and Methods Overall, 180 samples were used for polymerization shrinkage (buoyancy and optical methods) and degree of conversion tests in which they were divided into Group 1, nanofilled composite (Filtek-Z350-XT; 3M ESPE, St Paul, MN 55144-1000, USA), Group 2, microhybrid composite (Zmack-Comp), and Group 3 , nanohybrid composite ( $\mathrm{Zr}$-Hybrid). Polymerization shrinkage test was performed using buoyancy and optical methods. For buoyancy method, samples were weighed in air and water to calculate the shrinkage value, whereas, for optical method, images of nonpolymerized samples were captured under a digital microscope and recaptured again after light-cured to calculate the percentage of shrinkage. Degree of conversion was tested using Fourier-transform infrared spectroscopy spectrometer.

Statistical Analysis Data were analyzed using one-way analysis of variance complemented by post hoc Dunnett's T3 test for polymerization shrinkage and Tukey's honestly significant difference test for degree of conversion. Level of significance was set at $p<0.05$.

Results Group 3 demonstrated similar polymerization shrinkage with Group 1, but lower shrinkage $(p<0.05)$ than Group 2 based on buoyancy method. However, optical method $(p<0.05)$ showed that Group 3 had the lowest shrinkage, followed by Group 1 and lastly Group 2 . Besides, Group 3 showed a significantly higher degree of conversion $(p<0.05)$ than Group 1 and comparable conversion value with Group 2.

Conclusions Zirconia-reinforced rice husk nanohybrid composite showed excellent shrinkage and conversion values, hence can be considered as an alternative to commercially available composite resins.
DOI https://doi.org/

$10.1055 / \mathrm{s}-0040-1713951$

ISSN 1305-7456.
License terms

() (1) $\ominus \circledast$ 


\section{Introduction}

Composite resin has been introduced since the late 1950s and is widely used as a restorative material in dentistry. ${ }^{1}$ It has several advantages over amalgam such as better aesthetic properties, ability to bond to the tooth structure, and allowing for better conservation of tooth structure. ${ }^{2}$ Due to all these advantages, the use of composite resin has increased drastically in modern preventive and restorative dentistry.

Despite the continuous advancements in composite resins, it still suffers from polymerization shrinkage that ranges from 2 to $5 \%{ }^{3}$ Polymerization shrinkage of composite resins occurs mostly due to the conversion of monomer into polymer chain in which the van der Waals forces are replaced by covalent bonds that pull the particles closer. ${ }^{2}$ This leads to interfacial polymerization stresses, causing gap formation at the dentine-bond interface, increasing the risk of recurrent caries and consequently restoration failures. ${ }^{4,5}$ Several methods have been described to reduce polymerization shrinkage such as using incremental placement of composite resin, soft start light-curing technique, stress absorbing liners, use of composite resin with higher filler content, or modifying the resin component in composite resin., ${ }^{2,46}$ The degree of conversion also has a major impact on the success of composite resin restorations. ${ }^{7}$ The physical and mechanical properties of composite resins are directly influenced by the level of conversion during polymerization. ${ }^{8}$ The degree of conversion is determined by the proportion of remaining aliphatic $\mathrm{C}=\mathrm{C}$ double bonds in a polymerized sample to the total number of $\mathrm{C}=\mathrm{C}$ bonds in the nonpolymerized sample. Ideally, a composite resin should have all its monomer converted into polymer. A lower degree of conversion will cause a reduction in binding of polymer network and lead to a decrease in polymerization shrinkage. ${ }^{9}$ To date, many advanced techniques have been used to determine the degree of conversion of composite resins among which Fourier-transform infrared spectroscopy (FTIR) is the most widely used technique. ${ }^{8}$

Due to global environmental concern, silica from natural resources has started to raise interest among researchers to reinforce and improve the mechanical properties of composite and rubber materials. ${ }^{10,11}$ As a result, a new version of nanohybrid composite resin using rice husk as silica filler has been introduced. ${ }^{12}$ Rice husk is considered as an agricultural biowaste and the advantages of using such natural product in dental composites are ascribed to less cost, decreased toxicity, greater sustainability, lighter weight, and being more environmentally friendly. ${ }^{12}$ However, information regarding the use of rice husk as silica in dental composite is still lack in the literature. ${ }^{12,13}$ Previous study found out that rice husk nanohybrid composite demonstrated slightly inferior physical and mechanical properties than commercialized microhybrid composite ( $Z$ 250), but these values were still within the acceptable range. ${ }^{12}$

Recently, zirconia, also known as zirconium dioxide, which is a white crystalline oxide of zirconium, has been widely used in the field of dentistry due to its excellent physical and mechanical strength, biocompatibility, and optical properties, since zirconia can backscatter incident light and exhibit minimal light absorption from electronic polarization, therefore, giving it a translucent appearance that enhances its similarity with tooth dentine. ${ }^{14,15}$ Considering a more innovative approach, the present study added $10 \%$ $\mathrm{w} / \mathrm{w}$ zirconia nanopowder into this rice husk nanohybrid composite resin to reinforce the material. Hence, one can speculate that this new nanohybrid composite resin is able to demonstrate better physical, mechanical, and optical properties. To the extent of our knowledge, studies on the shrinkage and degree of conversion of this new zirconia-reinforced nanohybrid composite resin are not found in any published literature.

Thus, our objective for the current study was to compare the polymerization shrinkage and degree of conversion of this new zirconia-reinforced nanohybrid composite resin using organic filler derived from rice husk with other commercialized composite resins. The null hypothesis was that no significant difference exists in terms of polymerization shrinkage and degree of conversion between zirconia-reinforced rice husk nanohybrid composite and the other two commercially available composite resins.

\section{Materials and Methods}

\section{Preparation of Zirconia-Reinforced Nanohybrid Composite}

The filler-to-monomer ratio was 75:25. Resin matrix was composed of $50 \% \mathrm{w} / \mathrm{w}$ of bisphenol-A glycidyl methacrylate (bis-GMA), $48 \% \mathrm{w} / \mathrm{w}$ of triethylene glycol dimethacrylate (TEGDMA), and $2 \% \mathrm{w} / \mathrm{w}$ of camphorquinone (CQ). They were weighed using digital weighing scales (WN-FAN; Worner Laboratory or OEM, Zhejiang, China) with an accuracy of $0.0001 \mathrm{~g}$. The mixing of these chemicals was done in a mixing beaker placed on a vortex mixer (Fisherbrand Analog Vortex Mixer; Fisher Scientific International, Inc., Hampton, United States). The beaker was covered with aluminum foil to protect the resin from ambient light. The matrix was then stored in a refrigerator for 48 hours to stabilize it before mixing with filler component. After 48 hours, $25 \% \mathrm{w} / \mathrm{w}$ of the prepared matrix mixture was mixed using the same vortex mixer with $65 \% \mathrm{w} / \mathrm{w}$ of spherical nano-silica from rice husk obtained using sol-gel method from a previous study conducted in Universiti Sains Malaysia. ${ }^{12}$ Lastly, $10 \% \mathrm{w} / \mathrm{w}$ of pure zirconia nanopowder (MFCD00011310, American Elements, Los Angeles, United States) was added to form a homogenous composite material and the final composite product was kept in the refrigerator for further usage.

The filler volume $(V)$ was calculated with the following equation:

$$
V=\frac{m_{1}-m_{2}}{p_{1}-p_{2}} \times 100 \%
$$

Whereby, $m_{1}$ is the total weight of composite resin in gram, $\mathrm{g} ; m_{2}$ is the total weight of resin monomer used; $p_{1}$ is the total density of composite, which is $1.981 \mathrm{~g} / \mathrm{cm}^{3}$; and $p_{2}$ is the density of resin monomer used in $\mathrm{g} / \mathrm{cm}^{3}$. 
The information about resin monomer density was provided by the manufacturers: Bis-GMA (1.161 $\left.\mathrm{g} / \mathrm{cm}^{3}\right)$, TEGDMA $\left(1.090 \mathrm{~g} / \mathrm{cm}^{3}\right)$, and CQ $\left(1.006 \mathrm{~g} / \mathrm{cm}^{3}\right)$; thus, the filler volume of zirconia-reinforced rice husk nanohybrid composite resin obtained in the present study was 55.9\%.

\section{Grouping of Samples}

A total of 180 samples were prepared of which 120 samples were used for polymerization shrinkage tests (buoyancy and optical methods; each consisted of 60 samples) and the remaining 60 samples for the degree of conversion test. The total sample size was calculated based on $\mathrm{G}^{*}$ Power 3.1.9.4 software (Franz Faul; Universität Kiel, Germany) with a power of 0.8 added with $5 \%$ of dropout. Further, 60 samples corresponding to each test were subdivided into groups of 20 samples each according to the types of composite as below:

- Group 1-Nanofilled composite (Filtek-Z350-XT)

- Group 2-Microhybrid composite (Zmack-Comp)

- Group 3-Zirconia-reinforced rice husk nanohybrid composite (Zr-Hybrid)

The characteristics of each type of composite resin used are shown in - Table $\mathbf{1}$.

\section{Polymerization Shrinkage Testing Buoyancy Method}

The test was performed at room temperature using two digital weighing scales (WN-FAN; Worner Laboratory or OEM, Zhejiang, China) with an accuracy of $0.0001 \mathrm{~g}$. One digital scale was used to measure the mass of sample in air, whereas another scale was used to measure the mass of sample in water. A beaker filled with $100 \mathrm{~mL}$ of distilled water was placed on the digital scale that was used to measure the mass of sample in water. Each sample consisted of $1 \mathrm{~g}$ nonpolymerized composite resin. The test was conducted as recommended by the American Society for Testing and Materials: D792-13 specification. ${ }^{16}$ The nonpolymerized sample was wrapped with waterproof transparent plastic stretch film (Manual Stretch Film, Scientex, Shah Alam, Malaysia; -Fig. 1A) and compressed manually in between two flat glass plates (GLP2X2; United Scientific Supplies, Inc., Waukegan, United States) at a force of $\sim 2 \mathrm{~kg}$ measured with a metal weight (abs-sl-weightset-small; PCS Instruments, United Kingdom) resulting in a thin disc of sample of $\pm 1.0 \mathrm{~mm}$ thickness measured using a digital caliper (19975; Shinwa Rules Co., Ltd., Japan) with an accuracy of $0.01 \mathrm{~mm}$ (-Fig. 1B). The glass plates and transparent films were then removed carefully. The sample was weighed in air (-Fig. 1C) and then placed in a beaker containing $100 \mathrm{~mL}$ of distilled water ( - Fig. 1D) to measure the mass of sample in water. The density of the nonpolymerized sample in $\mathrm{g} / \mathrm{cm}$ was calculated according to the equation:

$$
P=\frac{m_{\text {water }}}{m_{\text {water }}-m_{\text {air }}}\left(P_{\text {water }}-P_{\text {air }}\right)+P_{\text {air }}
$$

Whereby, $P$ is the density of the composite resin, $m_{\text {water }}$ is the weight in grams $(\mathrm{g})$ of the material in water, $m_{\text {air }}$ is the weight in grams $(\mathrm{g})$ of the composite resin in air, $P_{\text {water }}$ is the density of distilled water according to density table at room temperature, and $P_{\text {air }}$ is the density of air, which is $0.0012 \mathrm{~g} / \mathrm{cm}$.

Subsequently, the nonpolymerized sample was removed from the beaker and dried thoroughly with filter paper (Grade 1, Whatman, Maidstone, United Kingdom). The entire measuring processes were performed within 60 seconds to prevent the nonpolymerized sample from undergoing premature polymerization under natural light. Next, the sample was light-cured for polymerization using light-emitting diode (LED) light-curing unit (Elipar Free Light 2; 3M ESPE, St. Paul, Minnesota, United States) with a light intensity of $600 \mathrm{~mW} / \mathrm{cm}^{2}$. The distance between the light source and the sample was standardized by placing a $1 \mathrm{~mm}$ thick glass slide (GLP2X2; United Scientific Supplies, Inc., Waukegan, United States) in between the light source and sample. The tip of the light source was in contact with the glass plate during the light polymerization process. The LED light-cure unit was calibrated by an LED radiometer (Demetron; Kerr, Danbury, Connecticut, United States) before being used and after every three samples, to ensure that the output of emitting light source was standardized throughout the experiment. The polymerization process under light cure was performed for 40 seconds as recommended by the manufacturers. The same protocol was repeated to obtain the density for the polymerized samples, and the mass of each sample was calculated by subtracting the mass of the transparent plastic film from the mass of the whole assembly. The percentage change in volume $(V)$ after polymerization was calculated from the densities according to the equation as shown in a similar study: ${ }^{17}$

$$
V=\left(\frac{1}{P_{\text {cured }}}-\frac{1}{P_{\text {un cured }}}\right) \frac{1}{P_{\text {un cured }}} \times 100
$$

Table 1 Characteristics of composite resins used in this study

\begin{tabular}{|l|l|l|l|l|l|}
\hline $\begin{array}{l}\text { Type of } \\
\text { composite }\end{array}$ & Name & Manufacturer & Type of resin monomer & Type of filler & Filler wt./vol. \\
\hline Nanofilled & Filtek Z350 XT & 3 M ESPE & $\begin{array}{l}\text { Bisphenol A glycidyl methacrylate (bis-GMA); } \\
\text { ethoxylated bisphenol A glycol dimethacrylate } \\
\text { (bis-EMA); urethane dimethacrylate (UDMA); } \\
\text { triethylene glycol dimethacrylate (TEGDMA); } \\
\text { polyethylene glycol dimethacrylate (PEGDMA) }\end{array}$ & $\begin{array}{l}\text { Zirconia and } \\
\text { silica }\end{array}$ & $78.5 \% / 63.3 \%$ \\
\hline Microhybrid & Zmack Comp & Zhermack & Bis-GMA; bis-EMA; TEGDMA & $\begin{array}{l}\text { Bariumglass and } \\
\text { silica }\end{array}$ & $\begin{array}{l}\text { Zirconia and rice } \\
\text { husk silica }\end{array}$ \\
\hline Nanohybrid & Zr-Hybrid & $\begin{array}{l}\text { Universiti Sains } \\
\text { Malaysia }\end{array}$ & Bis-GMA; TEGDMA & $75 \% / 55.9 \%$ \\
\hline
\end{tabular}




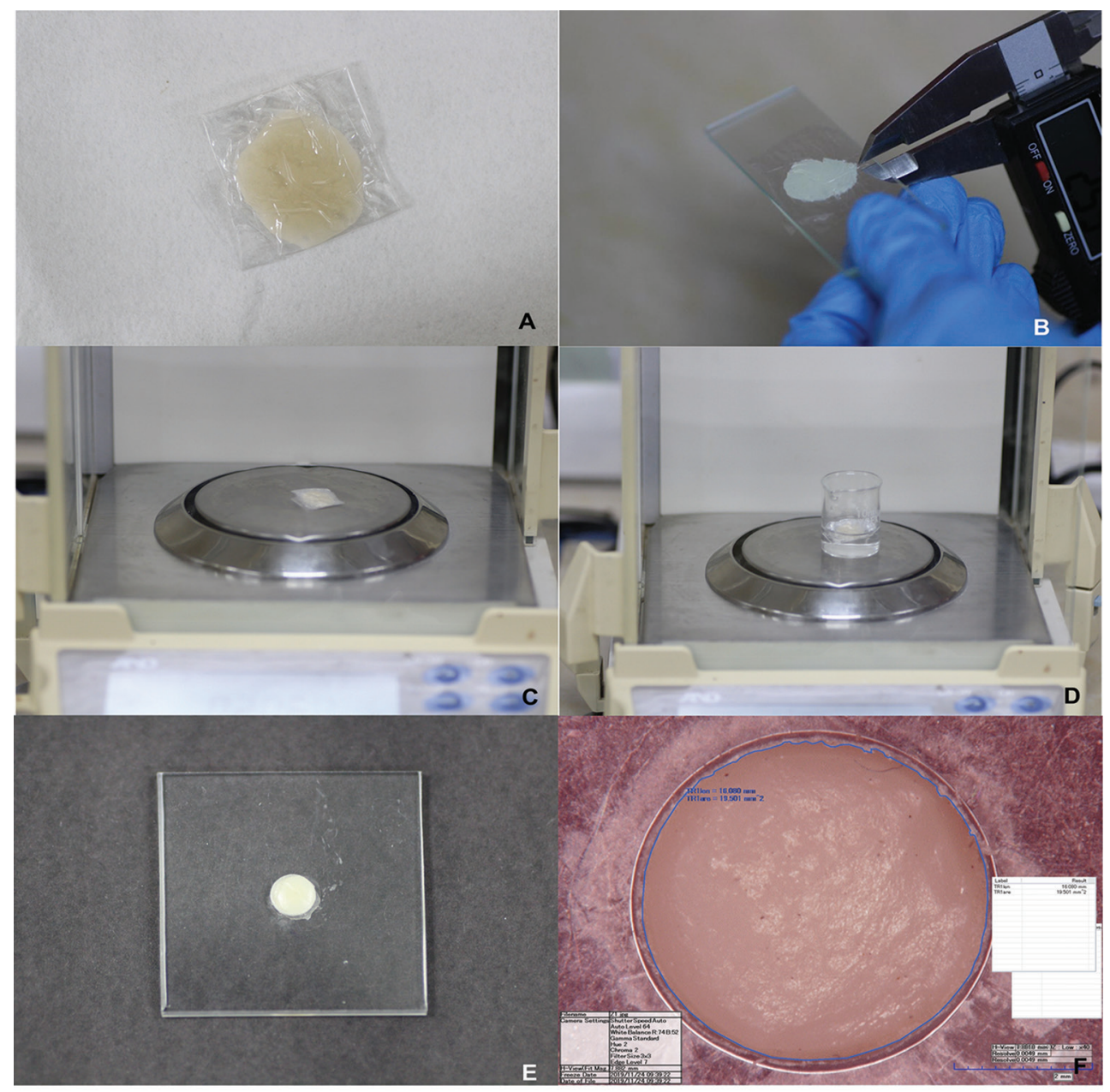

Fig. 1 (A) Nonpolymerized sample wrapped with waterproof transparent plastic stretch film. (B) Thickness of nonpolymerized sample was measured using a digital caliper with accuracy of $0.01 \mathrm{~mm}$ to ensure a thin disc about $\pm 1.0 \mathrm{~mm}$. (C) Mass in air of nonpolymerized sample weighed using a digital weighing scale. (D) Mass in water of nonpolymerized sample weighed in beaker containing $100 \mathrm{~mL}$ distilled water using a digital weighing scale. (E) Nonpolymerized sample placed on a round plastic disc with diameter of $10 \mathrm{~mm}$ and thickness of $2 \mathrm{~mm}$. (F) Sample was measured under Hirox 3D Digital microscope using optical method.

\section{Optical Method}

Composite samples from each group were placed on a smooth round plastic disc with a diameter of $10 \mathrm{~mm}$ and a thickness of $2 \mathrm{~mm}$ (-Fig. 1E). Then, the plastic disc was covered by two glass plates (GLP2X2) and compressed manually at a force of $\sim 2 \mathrm{~kg}$ measuring with a metal weight (abs-sl-weight-set-small) to ensure a flat surface and to remove excess material. The glass plates were removed carefully, and a prepolymerization image was captured under a digital microscope (Hirox 3D Digital microscope; RH-2000, Hirox Co Ltd., Tokyo, Japan) at a 20x magnification. The surface area $\left(S_{1}\right)$ and its thickness $\left(H_{1}\right)$ were traced ( - Fig. 1F) and measured by two blinded examiners using Advanced 3D Hirox software for volume and area measurements. The volume $\left(V_{1}\right)$ of the nonpolymerized sample was obtained by:

Volume, $V\left(\mathrm{~mm}^{3}\right)=S \times H$, whereby $S$ is the surface area and $H$ is the thickness.
The sample then underwent polymerization for 40 seconds with the same light-curing unit using identical polymerization protocol as mentioned in previous buoyancy method. Postpolymerization image was recaptured after the polymerization process completed. The surface area $\left(S_{2}\right)$ and thickness $\left(\mathrm{H}_{2}\right)$ of the polymerized sample were traced and measured. The volume $\left(V_{2}\right)$ was calculated as above. Percentage of shrinkage was then calculated based on the equation:

$$
\text { Shrinkage }=\left(V_{2}-V_{1}\right) / V_{1} \times 100 \%
$$

\section{Degree of Conversion Test}

Nonpolymerized composite samples weighing $0.5 \mathrm{~g}$ from each group were wrapped with the waterproof transparent plastic stretch film (Manual Stretch Film; Scientex, Shah Alam, Malaysia) and compressed between two glass plates (GLP2X2) with the same $\sim 2 \mathrm{~kg}$ metal weight used in shrinkage study until the sample was $\sim 1 \mathrm{~mm}$ in thickness measured using the same digital caliper. The glass plates 
and transparent films were gently and carefully removed. An FTIR spectrometer (Nicolet Summit FTIR Spectrometer; Thermo Fisher Scientific, Massachusetts, United States) operating under the conditions of $600-4,000 \mathrm{~cm}^{-1}$ wavelength, $4 \mathrm{~cm}$ resolution, and 32 scans co-addition was used to test the nonpolymerized sample. After that, the polymerization process was performed using the same LED light-curing unit as mentioned in earlier standardized polymerization protocol. The polymerized sample was tested again with the same FTIR spectrometer. The percentage of unreactive carbon-carbon double bonds $(\% \mathrm{C}=\mathrm{C})$ was determined from the ratio of the absorbance intensities of aliphatic $\mathrm{C}=\mathrm{C}$ (peak at $1,638 \mathrm{~cm}$ ) against the internal standard aromatic $\mathrm{C}-\mathrm{C}$ (peak at $1,608 \mathrm{~cm}$ ). Thus, the degree of conversion was obtained by:

$$
\text { Degree of Conversion }=\left(\frac{\frac{P_{1}}{P_{2}} \text { (cured) }}{\left.\frac{P_{1}}{P_{2}} \text { (Uncured) }\right)} \times 100 \%\right. \text {, }
$$

whereby $P_{1}=$ peak area at $1,638 \mathrm{~cm}^{-1}$ and $P_{2}=$ peak area at $1,608 \mathrm{~cm}$.

\section{Statistical Analysis}

Statistics were performed using the Statistical Package for the Social Sciences (IBM, New York, New York, United States) version 24 for Windows 10.0. The level of statistical significance was set at 0.05 . The data passed the normality test according to Shapiro-Wilk test. Data analysis for polymerization shrinkage using both buoyancy and optical methods was performed using one-way analysis of variance (ANOVA) complemented by post hoc Dunnett's T3 multiple comparison test, whereas data analysis for degree of conversion was performed using one-way ANOVA complemented by post hoc Tukey's honestly significant difference (HSD) multiple comparison test.

\section{Results}

The null hypothesis was partially rejected. Results from - Table 2 suggested that Group 1 and Group 3 exhibited significantly lower shrinkage value than Group 2 in both buoyancy $(p=0.001)$ and optical $(p=0.001)$ methods. Although Group 1 and Group 3 showed similar shrinkage value ( $p=1.000$ ) based on buoyancy method, Group 3 was found to have slightly lower shrinkage value $(p=0.864)$ than Group 1 based on optical method. Post hoc Dunnett's T3 test showed that there were significant differences between Group 1 and Group $2(p=0.007)$ and between Group 2 and Group 3 ( $p=0.013)$ using buoyancy method. A similar pattern was also noted in optical method whereby significant differences were noted between Group 1 and Group $2(p=$ 0.001 ) and between Group 2 and Group 3 ( $p=0.001$ ). Besides, analysis of correlation revealed that there was a statistically significant correlation between results obtained from buoyancy method and those from optical method $(p=0.029)$ based on Pearson Correlation Coefficient $(r=0.283)$, which suggested the reliability of both tests.

On the other hand, the present study revealed that Group 1 exhibited the lowest degree of conversion followed by Group 2 and lastly Group $3(p=0.001)$ based on - Table 3. Post hoc Tukey's HSD test showed that there were significant differences between Group 1 and Group $2(p=0.001)$ and between Group 1 and Group $3(p=0.001)$, but no significant difference was noted between Group 2 and Group 3 $(p=0.922)$. Furthermore, no significant correlation $(p=0.191)$ was observed between polymerization shrinkage and degree of conversion in the present study.

\section{Discussion}

Over the past few decades, composite resins have been extensively studied and greatly improved. ${ }^{18}$ Although composite resins exhibit excellent esthetic and mechanical properties with low cytotoxicity, they still have a major drawback, which is polymerization shrinkage. ${ }^{19}$ Several factors were found to cause shrinkage in composite resin, such as the size of filler, amount of filler particles, and the type of resin matrix used. ${ }^{19-22}$ The introduction of nanocomposites with optimal distribution of nano-sized filler particles has been shown to decrease the polymerization shrinkage of composite resin. ${ }^{23}$ The smaller the filler size, the larger the area-to-volume ratio

Table 2 Polymerization shrinkage (\%) of different composite resins (one-way ANOVA) and multiple comparisons of all groups (Dunnett's T3)

\begin{tabular}{|c|c|c|c|c|c|c|c|c|}
\hline \multirow[t]{2}{*}{ Group } & \multirow{2}{*}{$\begin{array}{l}\text { Type of } \\
\text { composite }\end{array}$} & \multirow[t]{2}{*}{ Mean (SD) } & \multirow[t]{2}{*}{$F(d f)$} & \multirow[t]{2}{*}{$p$-Value } & \multicolumn{4}{|c|}{ Multiple comparisons } \\
\hline & & & & & Groups & $\begin{array}{l}\text { Mean } \\
\text { difference }\end{array}$ & $\begin{array}{l}\text { Standard } \\
\text { error }\end{array}$ & $p$-Values \\
\hline \multicolumn{9}{|c|}{ Buoyancy method } \\
\hline 1 & Filtek Z350 XT & $1.95( \pm 0.20)$ & \multirow[t]{3}{*}{$8.47(2,57)$} & \multirow[t]{3}{*}{$0.001^{\mathrm{a}}$} & 1 vs. 2 & -0.2812 & 0.084 & $0.007^{a}$ \\
\hline 2 & Zmack Comp & $2.23( \pm 0.34)$ & & & 1 vs. 3 & -0.014 & 0.057 & 1.000 \\
\hline 3 & Zr-Hybrid & $1.95( \pm 0.16)$ & & & 2 vs. 3 & 0.267 & 0.087 & $0.013^{\mathrm{a}}$ \\
\hline \multicolumn{9}{|c|}{ Optical method } \\
\hline 1 & Filtek Z350 XT & $1.95( \pm 0.12)$ & \multirow[t]{3}{*}{$5.96(2,57)$} & \multirow[t]{3}{*}{$0.001^{\mathrm{a}}$} & 1 vs. 2 & -0.434 & 0.047 & $0.001^{\mathrm{a}}$ \\
\hline 2 & Zmack Comp & $2.39( \pm 0.17)$ & & & 1 vs. 3 & -0.082 & 0.034 & 0.864 \\
\hline 3 & Zr-Hybrid & $1.94( \pm 0.95)$ & & & 2 vs. 3 & 0.352 & 0.044 & $0.001^{\mathrm{a}}$ \\
\hline
\end{tabular}

Abbreviations: ANOVA, analysis of variance; SD, standard deviation.

aSignificance set at 0.05 . 
Table 3 Degree of conversion (\%) of different composite resins (one-way ANOVA) and multiple comparisons of all groups (Tukey's HSD)

\begin{tabular}{|c|c|c|c|c|c|c|c|c|}
\hline \multirow[t]{2}{*}{ Group } & \multirow{2}{*}{$\begin{array}{l}\text { Type of } \\
\text { composite }\end{array}$} & \multirow[t]{2}{*}{ Mean (SD) } & \multirow[t]{2}{*}{$F(d f)$} & \multirow[t]{2}{*}{$p$-Value } & \multicolumn{4}{|c|}{ Multiple comparison } \\
\hline & & & & & Groups & $\begin{array}{l}\text { Mean } \\
\text { difference }\end{array}$ & $\begin{array}{l}\text { Standard } \\
\text { error }\end{array}$ & $p$-Values \\
\hline 1 & Filtek Z350 XT & $67.37( \pm 4.34)$ & \multirow[t]{3}{*}{$12.91(2,57)$} & \multirow[t]{3}{*}{$0.001^{\mathrm{a}}$} & 1 vs. 2 & 8.873 & 1.495 & $0.001^{\mathrm{a}}$ \\
\hline 2 & Zmack Comp & $75.25( \pm 6.69)$ & & & 1 vs. 3 & 3.686 & 0.994 & $0.001^{\mathrm{a}}$ \\
\hline 3 & Zr-Hybrid & $75.61( \pm 5.28)$ & & & 2 vs. 3 & -5.187 & 1.181 & 0.922 \\
\hline
\end{tabular}

Abbreviations: ANOVA, analysis of variance; HSD, honestly significant difference; SD, standard deviation.

asignificance set at 0.05 .

of filler that can be incorporated in the resin matrix, and thus the reduced volumetric shrinkage. ${ }^{17}$ This explains the lower shrinkage value of Filtek-Z350-XT (nanofilled) and Zr-Hybrid (nanohybrid) as compared with Zmack-Comp (microhybrid) in the present study, which is in agreement with other similar studies. ${ }^{23-26}$ Other possible reasons that influence the shrinkage could be the filler loading and type of methacrylate monomers used. ${ }^{19}$

The amount of filler content not only plays an important role in mechanical properties and wear resistance, but also influences shrinkage stress of composite resin. ${ }^{27}$ Benefits of high filler content are improved mechanical and physical properties, decreased thermal expansion and contraction, and improved workability. ${ }^{27-30}$ On the contrary, lower filler content causes higher shrinkage in composite resin since filler particles do not mitigate the polymerization process. ${ }^{3,31}$ In addition, a lower filler volume indicates more space for the remaining resin matrix and increases the volume of polymerizable resin. ${ }^{26}$ The filler content in Zmack-Comp (77\% w/w; $57 \%$ vol.) is lesser than Filtek-Z350-XT (78.5\% w/w; 63.3\% vol.), which explained its higher shrinkage value. However, an unusual finding in the present study was noted in which Zr-Hybrid with the lowest filler content (75\% w/w; $55.9 \%$ vol.) showed lower shrinkage value than Zmack-Comp and Filtek-Z350-XT. The explanation could be based on the different types of monomer combinations used in $\mathrm{Zr}$-Hybrid and Zmack-Comp.

The type of monomer used greatly affects the polymerization shrinkage of composite resins. Using high-molecular-weight monomers with the presence of aromatic ring and fewer double bonds per unit of weight, such as bis-GMA and ethoxylated bisphenol-A dimethacrylate (bis-EMA), reduce the polymerization shrinkage. ${ }^{26}$ Nevertheless, high-molecular-weight monomers exhibit high viscosity. ${ }^{32}$ Therefore, low-molecular-weight monomers, such as TEGDMA and urethane dimethacrylate (UDMA), are added to dilute high-molecular-weight monomers and increase the resin mobility, but such addition increases polymerization shrinkage. ${ }^{32}$ All composite resins used in the present study contained bis-GMA and TEGDMA. The ratio of TEGDMA to bis-GMA in Zr-Hybrid is low, resulting in a lower shrinkage. ${ }^{19}$ In Filtek-Z350-XT, most of the TEGDMA was substituted by polyethylene glycol dimethacrylate (PEGDMA) and UDMA, which presumably caused a slight difference in shrinkage value when compared with Zr-Hybrid.

The degree of conversion is another relevant factor to be considered to evaluate composite resins because it strongly influences the mechanical properties and long-term degradation resistance. ${ }^{33} \mathrm{~A}$ high degree of conversion leads to excellent physical and mechanical properties enabling the restoration to withstand strong masticatory forces. ${ }^{30}$ However, a low degree of conversion not only compromises the physical and mechanical properties, but also exhibits a high amount of unreacted monomers that may irritate the soft tissues, stimulate the growth of bacteria, and cause allergic reactions. ${ }^{34}$ The degree of conversion is also associated with the amount of filler particles in composite resin, where the degree of conversion decreased with increased filler loading due to the restricted mobility of resin-monomers. ${ }^{30}$ In the present study, FTIR was used to determine the degree of conversion of composite resins and the result showed that nanofilled composite, Filtek-Z350-XT, yield the lowest conversion value (67.37\%). This is in line with previous studies that suggested that nanofilled composite resin demonstrated a lower degree of conversion. ${ }^{35,36}$ Although Zr-Hybrid (75.61\%) has the lowest filler content, it demonstrated a comparable degree of conversion with Zmack-Comp (75.25\%).

This could also be explained on the basis of the resin matrix component. Zmack-Comp showed slightly higher conversion level most probably due to the partial substitution of bis-GMA, a stiff and hydrogen-bonded molecule, with a longer and more flexible bis-EMA molecule. ${ }^{30}$ Apart from that, bis-EMA does not have strong secondary intermolecular interactions, which also explains toward a higher degree of conversion in Zmack-Comp as compared with Zr-Hybrid. ${ }^{37}$ TEGDMA has a lower concentration of hydrogen bonds to be disrupted; thus, it exhibits lower viscosity and higher rate of conversion. ${ }^{38}$ Additionally, TEGDMA has a lower molecular weight that facilitates packing and increases the chance of termination by disproportionation. This highly flexible molecule consisting of ether linkages in the backbone causes primary cyclization and increases the termination rate at higher conversion levels. ${ }^{39}$ Thus, Filtek-Z350-XT was reported to have a significantly lower degree of conversion 
due to the significant replacement of TEGDMA with UDMA and PEGDMA.

Previous studies have suggested a direct relationship between the degree of conversion and polymerization shrinkage. ${ }^{2,40,41} \mathrm{~A}$ higher degree of conversion will lead to a higher polymerization shrinkage and vice versa. This trend was found in Filtek-Z350-XT and Zmack-Comp based on the result of the present study in which Filtek-Z350-XT with lower shrinkage demonstrated lower degree of conversion, whereas Zmack-Comp with higher shrinkage showed higher degree of conversion. However, such a trend was not presented in Zr-Hybrid ( $p=0.191$ ) because Zr-Hybrid with lower shrinkage value demonstrated the highest degree of conversion, which is an odd and interesting finding.

Several limitations could be found in the present study. For instance, although FTIR is widely used to verify the degree of conversion of composite material, ${ }^{42}$ it does not permit the depth of cure assessment in which the differences in the polymerization reaction at different levels of the composite sample are ignored. Besides, even though buoyancy method in polymerization shrinkage test provides a better understanding of the entire three-dimensional volumetric change of composite resin, the presence of voids or entrapment of air bubbles in the composite specimen will cause incorrect measurements and greatly affect the result of the study. There is also a possibility that water molecules may enter the plastic stretch film and alter the polymerization reaction of composite resin. Nevertheless, optical method used in the present study only shows two-dimensional surface shrinkage of the composite material without providing more detailed information regarding the shrinkage direction. ${ }^{2}$ The accuracy of this method in visualizing internal defects or failures of the composite specimen is challenging. Thus, a higher sensitivity-measuring tool such as microcomputed tomography image scan is required to overcome such limitations and enhance the internal validity of the study. ${ }^{27}$ Apart from that, the results of the present study should be verified on a larger sample to provide a more reliable and valid outcome.

Within the limitation of this study, Zr-Hybrid was found to have lower shrinkage than Filtek-Z350-XT and Zmack-Comp with a significantly higher degree of conversion than FiltekZ350-XT and comparable conversion value with Zmack-Comp. Further studies are suggested to improve the shrinkage value and conversion rate of $\mathrm{Zr}$-Hybrid by manipulating the filler content and type of monomers used. Besides, comparisons between the polymerization shrinkage and degree of conversion of Zr-Hybrid with other advanced resin-based composites, such as low-shrinkage and bulk-filled composite materials, are warranted to determine its clinical relevance.

\section{Clinical Relevance}

An ideal composite resin should exhibit low shrinkage and high degree of conversion to imply long-term success of the restoration clinically.
Present new zirconia-reinforced rice husk nanohybrid composite showed excellent shrinkage and conversion value than commercialized composite resins.

\section{Authors' Contributions}

G.S.S.L. designed the study, performed the experiments, and prepared the manuscript. N.R.N.A.G. consulted and performed statistical evaluation, and contributed substantially to the discussion. N.H.I. assisted in sample preparation and contributed substantially to the discussion. K.P.S. designed the study, contributed substantially to the discussion, and proofread the manuscript. N.M.M.Y. assisted in statistical analysis and contributed substantially to the discussion.

\section{Funding}

The authors received financial support from Universiti Sains Malaysia (USM) research university grant scheme 1001/PPSG/8012215, short-term grant scheme 304/PPSG/ 6316456, and USM Fellowship Scheme.

\section{Conflict of Interest}

None declared.

\section{Acknowledgments}

The authors would like to thank Mr Baharuddin Bin Zakaria for helping in FTIR processing, and Dr Arif Awang Nawi for assistance in statistical analysis. Furthermore, we would also like to thank the management of USM, Kubang Kerian, Kelantan, for granting the permission to the investigators to use space and assets belonging to the hospital during the process of conducting this research.

\section{References}

1 Schneider LF, Cavalcante LM, Silikas N. Shrinkage stresses generated during resin-composite applications: a review. J Dent Biomech 2010;2010. Doi: 10.4061/2010/131630

2 Kaisarly D, Gezawi ME. Polymerization shrinkage assessment of dental resin composites: a literature review. Odontology 2016;104(3):257-270

3 Braga RR, Ballester RY, Ferracane JL. Factors involved in the development of polymerization shrinkage stress in resin-composites: a systematic review. Dent Mater 2005;21(10):962-970

4 Al Sunbul H, Silikas N, Watts DC. Polymerization shrinkage kinetics and shrinkage-stress in dental resin-composites. Dent Mater 2016;32(8):998-1006

5 Canellas TA, de Almeida Neves A, Dos Santos IKB, de Rezende ARP, Fellows CE, da Silva EM. Characterization of low-shrinkage dental composites containing methacrylethyl-polyhedral oligomeric silsesquioxane (ME-POSS) J Mech Behav Biomed Mater 2019;90:566-574

6 Marchesi G, Breschi L, Antoniolli F. Di Lenarda R, Ferracane J, Cadenaro M. Contraction stress of low-shrinkage composite materials assessed with different testing systems. Dent Mater 2010;26(10):947-953

7 Tarle Z, Attin T, Marovic D, Andermatt L, Ristic M, Tauböck TT. Influence of irradiation time on subsurface degree of conversion and microhardness of high-viscosity bulk-fill resin composites. Clin Oral Investig 2015;19(4):831-840 
8 Moraes LG, Rocha RS, Menegazzo LM, de Araújo EB, Yukimito $\mathrm{K}$, Moraes JC. Infrared spectroscopy: a tool for determination of the degree of conversion in dental composites. J Appl Oral Sci 2008;16(2):145-149

9 Nagem Filho H, Nagem HD, Francisconi PA, Franco EB, Mondelli $\mathrm{RF}$, Coutinho KQ. Volumetric polymerization shrinkage of contemporary composite resins. J Appl Oral Sci 2007;15(5): 448-452

10 Siriwardena S, Ismail H, Ishiaku US, Perera MCS. Mechanical and morphological properties of white rice husk ash filled polypropylene/ethylene-propylene-diene terpolymer thermoplastic elastomer composites. J Appl Polym Sci 2002;85(2): 438-453

11 Ahmed K, Nizami SS, Riza NZ. Reinforcement of natural rubber hybrid composites based on marble sludge/Silica and marble sludge/rice husk derived silica. J Adv Res 2014;5(2):165-173

12 Noushad M, Ab Rahman I, Husein A, Mohamad D. Nanohybrid dental composite using silica from biomass waste. Powder Technol 2016;299:19-25

13 Yusoff NM, Johari Y, Ab Rahman I, et al. Physical and mechanical properties of flowable composite incorporated with nanohybrid silica synthesised from rice husk. J Mater Res Technol. 2019;8(3):2777-2785

14 Shahmiri R, Standard OC, Hart JN, Sorrell CC. Optical properties of zirconia ceramics for esthetic dental restorations: a systematic review. J Prosthet Dent 2018;119(1):36-46

15 Gallina BL, Busato MCA, Sicoli EA, Camilotti V, Mendonca MJ. Aged translucent aesthetic zirconia: bond strength analysis. Eur J Dent 2019;13(1):5-10

16 std . ASTM D792-13, Standard Test Methods for Density and Specific Gravity (Relative Density) of Plastics by Displacement. In: ASTM International, West Conshohocken, PA, 2013;2013

17 de Melo Monteiro GQ, Montes MA, Rolim TV, et al. Alternative methods for determining shrinkage in restorative resin composites. Dent Mater 2011;27(8):e176-e185

18 Cisneros AZ, Armas AS. A comparative study of bulk-fill composites: degree of conversion, post-gel shrinkage and cytotoxicity. Braz Oral Res 2019;33:e003

19 Gonçalves F, Azevedo CL, Ferracane JL, Braga RR. BisGMA/ TEGDMA ratio and filler content effects on shrinkage stress. Dent Mater 2011;27(6):520-526

20 Amirouche-Korichi A, Mouzali M, Watts DC. Effects of monomer ratios and highly radiopaque fillers on degree of conversion and shrinkage-strain of dental resin composites. Dent Mater 2009;25(11):1411-1418

21 Barretto IC, Pontes LF, Carneiro KK, Araujo JL, Ballester RY, Silva $\mathrm{CM}$. Comparative analysis of polymerization shrinkage of different resin composites. Gen Dent 2015;63(2):41-45

22 Ellakwa A, Cho N, Lee IB. The effect of resin matrix composition on the polymerization shrinkage and rheological properties of experimental dental composites. Dent Mater 2007;23(10):1229-1235

23 Pereira RA, Araujo PA, Castañeda-Espinosa JC, Mondelli RF. Comparative analysis of the shrinkage stress of composite resins. J Appl Oral Sci 2008;16(1):30-34

24 Fugolin AP, Correr-Sobrinho L, Correr AB, Sinhoreti MA, Guiraldo RD, Consani S. Influence of irradiance on Knoop hardness, degree of conversion, and polymerization shrinkage of nanofilled and microhybrid composite resins. Gen Dent 2016;64(2):26-31
25 Frascino S, Fagundes TC, Silva U, et al. Randomized prospective clinical trial of class II restorations using low-shrinkage flowable resin composite. Oper Dent 2020;45(1):19-29

26 Alomari Q, Ajlouni R, Omar R. Managing the polymerization shrinkage of resin composite restorations: a review. SADJ 2007;62(1):12, 14, 16 passim

27 Soares CJ, Faria-E-Silva AL, Rodrigues MP, et al. Polymerization shrinkage stress of composite resins and resin cements - what do we need to know? Braz Oral Res 2017;31(suppl 1) :e62

28 Lin-Gibson S, Sung L, Forster AM, Hu H, Cheng Y, Lin NJ. Effects of filler type and content on mechanical properties of photopolymerizable composites measured across two-dimensional combinatorial arrays. Acta Biomater 2009;5(6):2084-2094

29 Cheng L, Zhang K, Weir MD, Melo MA, Zhou X, Xu HH. Nanotechnology strategies for antibacterial and remineralizing composites and adhesives to tackle dental caries. Nanomedicine (Lond) 2015;10(4):627-641

30 Rastelli AN, Jacomassi DP, Faloni AP, et al. The filler content of the dental composite resins and their influence on different properties. Microsc Res Tech 2012;75(6):758-765

31 AlShaafi MM. Factors affecting polymerization of resin-based composites: a literature review. Saudi Dent J 2017;29(2):48-58

32 Kwon YH, Jeon GH, Jang CM, Seol HJ, Kim HI. Evaluation of polymerization of light-curing hybrid composite resins. J Biomed Mater Res B Appl Biomater 2006;76(1):106-113

33 Rodrigues SA Jr, Scherrer SS, Ferracane JL, Della Bona A. Microstructural characterization and fracture behavior of a microhybrid and a nanofill composite. Dent Mater 2008; 24(9):1281-1288

34 Sideridou ID, Achilias DS. Elution study of unreacted Bis-GMA, TEGDMA, UDMA, and Bis-EMA from light-cured dental resins and resin composites using HPLC. J Biomed Mater Res B Appl Biomater 2005;74(1):617-626

35 Gonçalves F, Campos LMP, Rodrigues-Júnior EC, et al. A comparative study of bulk-fill composites: degree of conversion, post-gel shrinkage and cytotoxicity. Braz Oral Res 2018;32:e17

36 Ribeiro BC, Boaventura JM, Brito-Gonçalves Jd, Rastelli AN, Bagnato VS, Saad JR. Degree of conversion of nanofilled and microhybrid composite resins photo-activated by different generations of LEDs. J Appl Oral Sci 2012;20(2):212-217

37 Lemon MT, Jones MS, Stansbury JW. Hydrogen bonding interactions in methacrylate monomers and polymers. J Biomed Mater Res A 2007;83(3):734-746

38 Gonçalves F, Kawano Y, Pfeifer C, Stansbury JW, Braga RR. Influence of BisGMA, TEGDMA, and BisEMA contents on viscosity, conversion, and flexural strength of experimental resins and composites. Eur J Oral Sci 2009;117(4):442-446

39 FloydCJ,Dickens SH. Networkstructure of Bis-GMA-andUDMAbased resin systems. Dent Mater 2006;22(12):1143-1149

40 Silikas N, Eliades G, Watts DC. Light intensity effects on resin-composite degree of conversion and shrinkage strain. Dent Mater 2000;16(4):292-296

41 Al-Ahdal K, Ilie N, Silikas N, Watts DC. Polymerization kinetics and impact of post polymerization on the degree of conversion of bulk-fill resin-composite at clinically relevant depth. Dent Mater 2015;31(10):1207-1213

42 Galvão MR, Caldas SG, Bagnato VS, de Souza Rastelli AN, de Andrade MF. Evaluation of degree of conversion and hardness of dental composites photo-activated with different light guide tips. Eur J Dent 2013;7(1):86-93 